Check for updates

Cite this: Chem. Sci., 2019, 10, 936

๑ All publication charges for this article have been paid for by the Royal Society of Chemistry

Received 13th September 2018 Accepted 4th November 2018

DOI: $10.1039 / \mathrm{c} 8 \mathrm{sc} 04083 d$

rsc.li/chemical-science

\section{Selective electrochemical functionalization of the graphene edge $\uparrow$}

\author{
Anur Yadav, (D) ab Rodrigo M. lost, (D) ab Tilmann J. Neubert, (D) abc Sema Baylan, ${ }^{a}$ \\ Thomas Schmid ${ }^{\text {ad }}$ and Kannan Balasubramanian (D) *abd
}

\begin{abstract}
We present a versatile and simple method using electrochemistry for the exclusive functionalization of the edge of a graphene monolayer with metal nanoparticles or polymeric amino groups. The attachment of metal nanoparticles allows us to exploit surface-enhanced Raman scattering to characterize the chemistry of both the pristine and the functionalized graphene edge. For the pristine patterned graphene edge, we observe the typical edge-related modes, while for the functionalized graphene edge we identify the chemical structure of the functional layer by vibrational fingerprinting. The ability to obtain single selectively functionalized graphene edges routinely on an insulating substrate opens an avenue for exploring the effect of edge chemistry on graphene properties systematically.
\end{abstract}

\section{Introduction}

Graphene is the simplest stable atomically flat material that is being intensively investigated due to its unique physical and chemical properties. ${ }^{\mathbf{1 - 3}}$ The chemistry of the interface between graphene and its environment (gas or liquid) plays a crucial role in the characteristic properties. ${ }^{4-7}$ Hence, it is important to devise methods to modify this chemistry precisely as well as to understand the effect of the modification on the ensuing properties, to engineer graphene for an application of interest. ${ }^{8,9}$ Many methods for modification of graphene in its various forms are currently pursued. ${ }^{\mathbf{1 0 - 1 4}}$ As the surface dictates many properties of bulk materials, the edge of graphene is known to influence its electronic and chemical properties. The role played by the edges becomes dominant when narrowing down graphene sheets to nanoribbons, e.g. by lithographic patterning. ${ }^{15,16}$ The honeycomb lattice consists of different configurations of edges referred to as zigzag and armchair. Practically, however, combinations of both configurations are expected. The type of the edge determines the stability and the electronic structure. ${ }^{\mathbf{1 7 - 2 0}}$ Graphene edges have been characterized using electrochemistry, ${ }^{21}$ Raman spectroscopy, ${ }^{22,23}$ and at atomic resolution using scanning probe microscopy ${ }^{\mathbf{2 4}}$ and

\footnotetext{
${ }^{a}$ School of Analytical Sciences Adlershof (SALSA), Humboldt Universität zu Berlin, 10099 Berlin, Germany. E-mail: nano.anchem-at-hu-berlin.de

${ }^{b}$ Department of Chemistry, IRIS Adlershof, Humboldt Universität zu Berlin, 10099 Berlin, Germany

${ }^{c}$ Helmholtz-Zentrum Berlin für Materialien und Energie GmbH, Institut für SiliziumPhotovolatik, Kekuléstr. 5, 12489 Berlin, Germany

${ }^{d} B A M$, Federal Institute for Materials Research and Testing, Richard-Willstätter-Str. 11, 12489 Berlin, Germany

† Electronic supplementary information (ESI) available. See DOI: 10.1039/c8sc04083d
}

electron microscopy. ${ }^{25,26}$ Theoretically, the modification of the graphene edge is expected to modify the observed properties, such as oxygen groups modulating the electronic and magnetic properties of nanoribbons. ${ }^{20,27}$ Until now, most of the edge functionalization methods have been used on graphene flakes dispersed in solution, where a single object control and observation is not possible. ${ }^{28-32}$ There are two examples of chemical modification of the graphene edge carried out in the gas phase in ultrahigh vacuum at low temperatures. Using surface assisted coupling, tetrapyrroles were fused covalently to a graphene edge, ${ }^{33}$ while in another report individual tetrafluorotetracyanoquinodimethane molecules were fixed on the graphene edge by using an electronically inert molecular anchor. ${ }^{34}$ For both cases, the graphene edge was partially modified by using an elaborate and indirect methodology. The selective homogeneous modification of the edge of a single graphene sheet under ambient conditions has not been explored yet. Here we present a strategy to achieve this and at the same time gather spectroscopic information using the same construct, enabling us to corroborate the chemistry of the functional groups present or formed at the edge.

\section{Results and discussion}

Fig. 1(a-c) presents a scheme with the general procedure for the exclusive chemical functionalization of the graphene edge (GrEdge). CVD-graphene is first transferred to $\mathrm{Si} / \mathrm{SiO}_{2}$ substrates with pre-patterned electrodes using a wet transfer strategy. ${ }^{35}$ Polystyrene is used as a support polymer, which ensures minimal organic contamination. ${ }^{36}$ Following transfer, trace metal particles are removed using electrochemical etching (eetching). ${ }^{37}$ (Details of fabrication are presented in the experimental section, and in ESI Fig. S1 and S2 $\dagger$ ). Following transfer, 

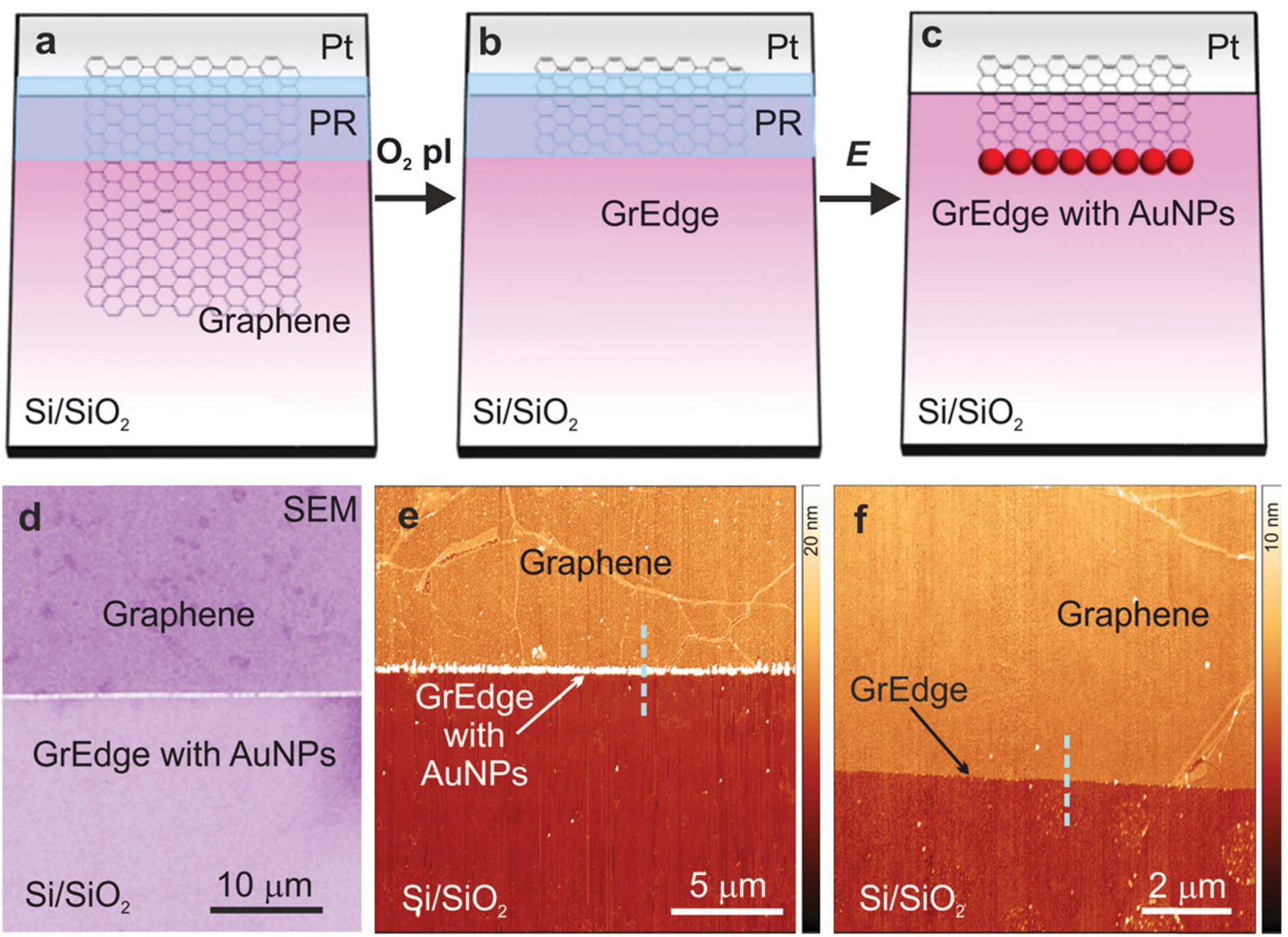

Fig. $1(a-c)$ Schematic of the procedure for fabrication and functionalization of a graphene edge (GrEdge): (a) patterning a photoresist layer (PR) on a graphene sheet on $\mathrm{Si} / \mathrm{SiO}_{2}$ with pre-patterned electrode (Pt). (b) Etching uncovered graphene regions with $\mathrm{O}_{2}$ plasma $\left(\mathrm{O}_{2} \mathrm{pl}\right)(\mathrm{c})$ electrodeposition of Au nanoparticles (AuNPs) at the GrEdge by application of a voltage (E) and dissolution of the photoresist. (d) Scanning electron microscope (SEM) image showing the modified GrEdge decorated with AuNPs. (e) AFM image of a GrEdge functionalized with AuNPs. (f) AFM image of a control sample with a non-functionalized edge. The cyan dashed lines have been used to extract line profiles shown in Fig. S5, ESI. $\dagger$

a positive photoresist is patterned using photolithography to define the location of the edge (Fig. 1(a)). The GrEdge is realized by removing the uncovered regions of the graphene sheet by a mild oxygen plasma treatment, leaving a neat exposed edge (Fig. 1(b)). The GrEdge is subsequently modified electrochemically using an appropriate precursor (Fig. 1(c)). The photoresist is an insulating layer of polymer that protects the graphene basal plane from coming in contact with the liquid, and ensures that a chemical reaction takes place exclusively at the GrEdge, which is the only part of the electrode exposed to the electrolyte solution. An absence of the photoresist will promote the electrochemistry reaction to occur at the whole surface of graphene and not only at the edge. ${ }^{38}$ The photoresist is finally removed in order to characterize the edge region. In the first example, the $\mathrm{Au}$ nanoparticles are deposited at the GrEdge through electrochemical reduction of a gold salt. Heterogeneous electron transfer between the gold species in solution $\left(\mathrm{AuCl}_{4}{ }^{-}\right)$and the GrEdge electrode results in the precipitation of gold at the edge. ${ }^{39}$ Specifically, a cathodic potential is applied (at the stage in Fig. 1(b)) in a solution of $\mathrm{KAuCl}_{4}$ (parameters in experimental section, and Fig. S3 in ESI $\dagger$ ). Fig. 1(d) shows a large-scale scanning electron microscopy image and Fig. 1(e) an atomic force microscopy (AFM) image of the edge region (after removal of photoresist), where it is clear that a chain of particles are generated exclusively at the graphene edge. Support that the particles are gold is obtained from X-ray spectroscopic analysis (ESI, Fig. S4†). As a control, Fig. 1(f) presents an AFM image of a GrEdge sample obtained by the same procedure without any edge functionalization. From line profiles in such AFM images (Fig. S5 $\dagger$ ) we infer that the unmodified samples shows a clean graphene surface (with a height of around $1 \mathrm{~nm}$ ), while the particles show an average height of $55 \pm 7 \mathrm{~nm}$. The particle size can in principle be varied by using different parameters for electrodeposition. ${ }^{\mathbf{4 0}}$

The deposition of AuNPs serves a twofold purpose. First, the exclusive deposition of particles at the edge can be confirmed by surface characterization as detailed above. Secondly, the presence of AuNPs at the edge allows us to perform selective spectroscopic characterization of the chemistry exclusively of the edge by virtue of surface enhanced Raman scattering (SERS). ${ }^{\mathbf{3 8 , 4 1}}$ Raman spectroscopy is widely used to investigate the electronic and chemical properties of graphene..$^{\mathbf{4 2 - 4 4}}$ It is sensitive to the nature of graphene defects and edges, and has been used to monitor doping effects. ${ }^{45}$ In our case, the presence of particles 
at the GrEdge results in a surface enhancement exclusively at the edge and in this manner, the observed SERS signals can be associated as coming predominantly from the edge. Another important advantage of using AuNPs is that it allows for local vibrational mapping by SERS, which has enabled us in the past to obtain information about functional groups on the graphene surface. ${ }^{\mathbf{4 6}}$ Hence, the construct can be directly used to investigate the presence or absence of functional groups at the patterned GrEdge.

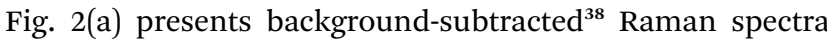
obtained at the basal plane, at the GrEdge without AuNPs and with AuNPs. Fig. 2(b) presents an AFM image of the GrEdgeAuNPs sample and the corresponding Raman maps (Fig. 2c, see Fig. S6 in ESI $\dagger$ for further analysis). For a better characterization of the edge, incident polarization was kept parallel to the edge, because the D-peak intensity strongly depends on the angle between incident polarization and edge. ${ }^{22}$ The Raman 2D map shows clearly that the intensity is relatively higher along the edge (indicative of SERS enhancement ${ }^{38,47}$ ), while the other Raman maps show that the disorder is exclusively observable at the edge.

The spectrum of the basal plane (black curve) in Fig. 2(a) shows as expected only $\mathrm{G}$ and $2 \mathrm{D}$ peaks, indicative of graphene of high quality with minimal defects. ${ }^{45}$ The edge spectrum without AuNPs (red curve) shows additionally a small D-peak and a miniscule shoulder $\left(\mathrm{D}^{\prime}\right.$-peak, $\sim 1620 \mathrm{~cm}^{-1}$ ) next to the G-peak. This is consistent with previous results, where both these signals are attributed to the presence of $\mathrm{sp}^{3}$-carbon, perturbing the honeycomb lattice and leading to an increase in the disorder-related $\mathrm{D}$ and $\mathrm{D}^{\prime}$ peaks of graphene. ${ }^{42,48-50}$ The $\mathrm{D}^{\prime}$ peak has been observed earlier on H-terminated zigzag graphene edges $^{22,23,51}$ and in graphane (hydrogenated graphene). ${ }^{52-54}$ Interestingly, in the Raman spectrum of GrEdge-AuNPs (SERS spectrum), again only the same 4 peaks are visible, with a difference only in the relative intensities. The increase in the intensity of D-peak may be partly attributed to resonant enhancement by plasmonic absorption of the AuNPs at this energy. ${ }^{55}$ It is worth mentioning that when AuNPs are deposited on the basal plane, the $\mathrm{D}^{\prime}$-peak does not appear. ${ }^{38}$

Reactive plasma treatment is considered a possible source of generation of oxygenated chemical groups. ${ }^{56}$ In order to verify if we are sensitive to oxygen-related functional groups, we have deliberately oxidized a GrEdge electrochemically ${ }^{57}$ and subsequently attached the nanoparticles. In this case, we can observe additional oxygen-related peaks in the SERS spectra (ESI, Fig. S7†). Such oxygen-related peaks are however absent in the SERS spectra of the pristine GrEdge (Fig. 2(a)) suggesting that we have a rather low density of oxygen-related functional groups here. This is most likely due to the very mild plasma conditions used. Stronger plasma conditions are not suited since this would affect the photoresist adversely. The absence of oxygenrelated groups at the edge has also been reported in other occasions, e.g. at the graphene edge under inert high vacuum conditions. ${ }^{25}$ SERS is known to be sensitive down to the single molecule level. ${ }^{47}$ It is worth mentioning that in the many 100 spectra (Fig. S8, ESI $\dagger$ ) that were measured on the pristine GrEdge with varying particle sizes, we could only observe the
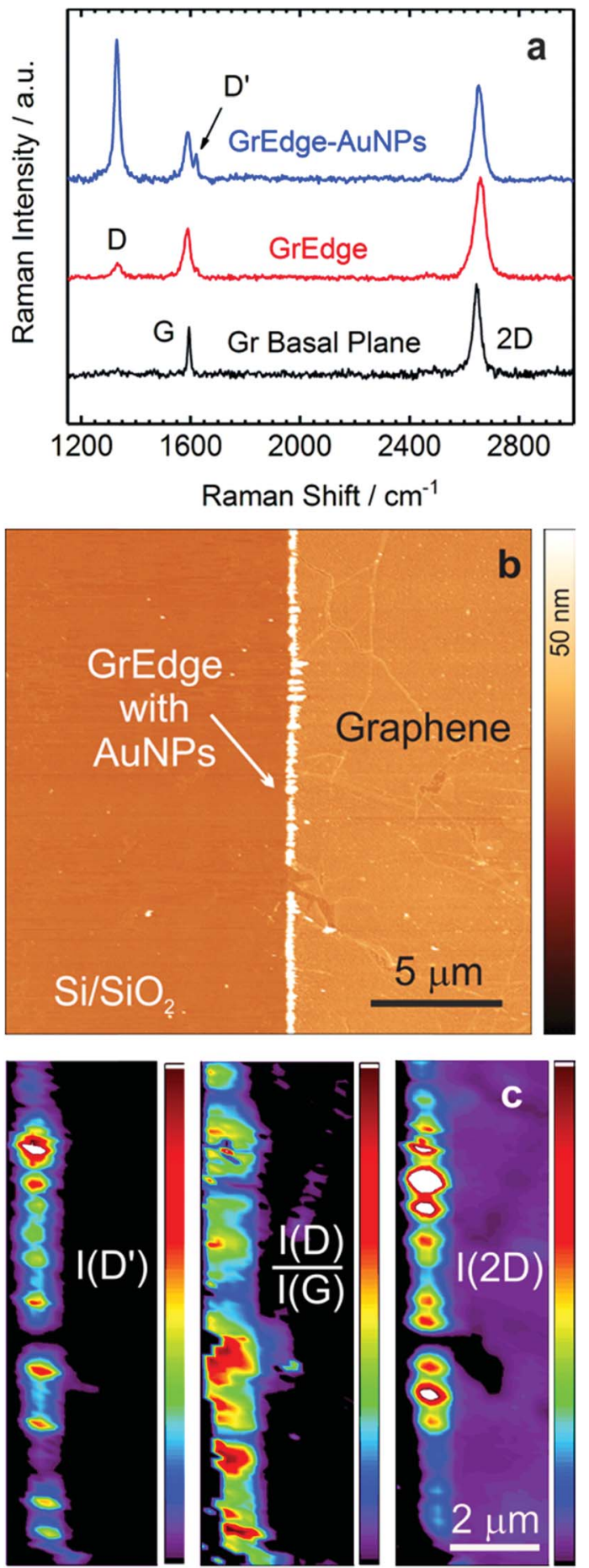

Fig. 2 (a) Comparison of Raman spectra from the graphene basal plane (black curve), the graphene edge (red curve) and the graphene edge with AuNPs (blue curve). (b) AFM image of a GrEdge with AuNPs and (c) the corresponding Raman map of $D^{\prime}\left(1620 \mathrm{~cm}^{-1}\right), D$ $\left(1330 \mathrm{~cm}^{-1}\right.$, relative to $\left.\mathrm{G} 1587 \mathrm{~cm}^{-1}\right)$, and $2 D\left(2653 \mathrm{~cm}^{-1}\right)$ intensities ( $\left.\lambda_{\text {ex }}: 633 \mathrm{~nm}, 4.5 \mathrm{~mW}, 1 \mathrm{~s}\right)$.

edge related graphene modes. Hence, we conclude that the patterned edge is most likely predominantly hydrogen terminated, with a rather low density of other functionalities.

As a second example, we demonstrate the functionalization of the GrEdge with aromatic amino groups through oxidative 
electropolymerization of 4-aminobenzylamine (ABA). Similar to AuNPs, the deposition of ABA is also driven by heterogeneous electron transfer reaction between the ABA species in solution and GrEdge electrode. The electron transfer reaction promotes the irreversible oxidation of 4-ABA with the formation of a polymer layer containing oligomers of varying chain length ${ }^{58}$ at the GrEdge. This is achieved by performing two cycles of cyclic voltammetry (Fig. S9 in ESI†) with GrEdge at the stage of Fig. 1(b) as the working electrode in an aqueous solution of ABA. In order to characterize the attached functional groups in the realized poly(aminobenzylamine) (pABA) layer, the GrEdgepABA is further modified with AuNPs using the same procedure as before. Since the polymer does not block electron transfer, it is still possible to reduce the gold species in solution. ${ }^{46} \mathrm{An}$ AFM image of a typical GrEdge-pABA-AuNPs sample is shown in Fig. 3(a), while Fig. 3(b) presents typical Raman spectra at the functionalized GrEdge before (red curve) and after (blue curve) attachment of AuNPs. The spectrum of GrEdge-pABA-AuNPs shows new peaks in addition to the four graphene-related peaks, which we attribute to the vibrational fingerprint of the pABA layer. The negligible change in $\mathrm{D}$ peak intensity in the red spectrum indicates that pABA is non-covalently attached to GrEdge. This is consistent with reports on the oxidative coupling of substituted anilines (such as ABA) resulting in a non-covalent attachment of the polymer on carbon nanotubes $^{39,59}$ and the graphene basal plane. ${ }^{46,60}$ This is also supported by the subsequent ability of the GrEdge-pABA to undergo electron transfer to obtain the AuNPs. By contrast, when the edge is functionalized with diazonium radicals (data not discussed here) - which is known to result in a covalent attachment - we were unable to obtain AuNPs at the GrEdge. This is consistent with covalently modified graphene showing much lower electron transfer rates. ${ }^{61}$

Table 1 presents a summary of the assigned Raman modes for the peaks observed commonly in the spectra recorded at many positions on several samples (Fig. S10, ESI $\dagger$ ). The Raman modes of pABA have some similarity with that of polyaniline due to their chemical structure..$^{46,62,63}$ The mode at $1632 \mathrm{~cm}^{-1}$ is assigned to $\mathrm{NH}_{2}$ deformation characteristic of primary amine group ${ }^{64}$ while the other modes at 1593 and $1566 \mathrm{~cm}^{-1}$ are due to $\mathrm{C}-\mathrm{C} / \mathrm{C}=\mathrm{C}$ ring stretching of the benzenoid form. ${ }^{65}$ The occurrence of two bands at 1358 and $1399 \mathrm{~cm}^{-1}$ is attributed to
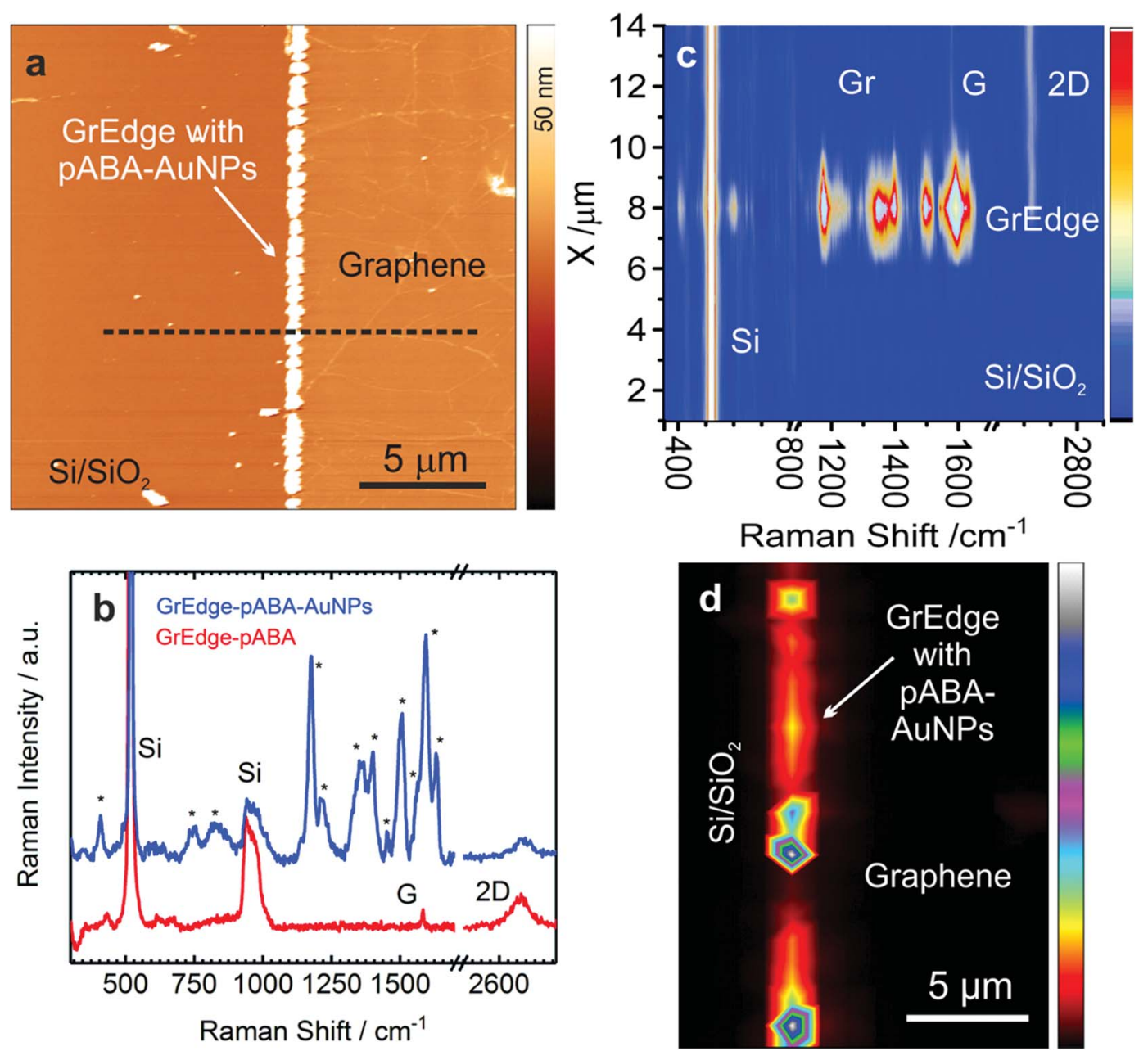

Fig. 3 (a) AFM image of a typical GrEdge functionalized with poly(aminobenzylamine) (pABA) and AuNPs, referred to as GrEdge-pABA-AuNPs. (b) Comparison of the Raman spectra at GrEdge-pABA (red curve) and GrEdge-pABA-AuNPs (blue curve). (c) Raman map along the line shown in (a). (d) Map of the $\mathrm{C}-\mathrm{N}$ stretching mode $\left(1358 \mathrm{~cm}^{-1}\right)$ of the same region as in (a) $\left(\lambda_{\text {ex }}: 633 \mathrm{~nm}, 3.4 \mathrm{~mW}, 2 \times 1 \mathrm{~s}\right)$. 
Table 1 Assignment of peaks in the spectra of GrEdge-pABA-AuNPs to the vibrational modes of poly(aminobenzylamine)

\begin{tabular}{ll}
\hline Raman shift $\left( \pm 2 \mathrm{~cm}^{-1}\right)$ & Assigned peaks \\
\hline 1632 & $\mathrm{NH}_{2}$ deformation \\
1593 & $\mathrm{C}=\mathrm{C}$ ring stretch \\
1566 & $\mathrm{C}-\mathrm{C}$ ring stretch \\
1504 & $\mathrm{C}-\mathrm{C}$ ring stretch \\
1453 & $\mathrm{CH}_{2}$ scissor vibration \\
1400 & $\mathrm{C}-\mathrm{N}$ stretch \\
1358 & $\mathrm{C}-\mathrm{N}$ stretch \\
1214 & $\mathrm{C}-\mathrm{C}-\mathrm{N}$ bending \\
1175 & $\mathrm{C}-\mathrm{H}$ bending \\
1137 & $\mathrm{C}-\mathrm{N}$ stretch \\
825 & $\mathrm{C}-\mathrm{H}$ wag \\
744 & $\mathrm{C}-\mathrm{N}$ out of plane deformation \\
409 & $\mathrm{C}-\mathrm{N}-\mathrm{C}$ bending \\
\end{tabular}

$\mathrm{C}-\mathrm{N}$ stretch. ${ }^{66}$ The mode at $1175 \mathrm{~cm}^{-1}$ is attributed to $\mathrm{C}-\mathrm{H}$ bending. The band at around $1504 \mathrm{~cm}^{-1}$, arises from aromatic $\mathrm{C}-\mathrm{C}$ stretching and a mode in low frequency region at $409 \mathrm{~cm}^{-1}$ is assigned to $\mathrm{C}-\mathrm{N}-\mathrm{C}$ bending of amines. The remaining modes at 1137 and $1214 \mathrm{~cm}^{-1}$, which are assigned to $\mathrm{C}-\mathrm{N}$ stretch and $\mathrm{C}-\mathrm{C}-\mathrm{N}$ bending, may arise due to the free $\mathrm{NH}_{2}$ group, which is not participating in polymerization reaction. ${ }^{64}$ These characteristics of the Raman spectra of pABA confirm its formation at the GrEdge. A Raman map along a cross-section across the edge is shown in Fig. 3(c), where it is apparent that the pABA modes are found exclusively at the edge. This is further confirmed by the Raman map of the $\mathrm{C}-\mathrm{N}$ stretching mode, as shown in Fig. 3(d). The spots corresponding to the vibrational modes of the pABA layer are elongated since the signals are convoluted by the diffraction-limited spot and the size of the nanoparticles.

\section{Conclusions}

In conclusion, we have demonstrated a versatile strategy for the realization of single functionalized graphene edges by selective and homogeneous non-covalent modification with Au nanoparticles or aromatic amino groups under ambient conditions on an insulating substrate. The presence of aromatic amino groups at the modified GrEdge could be corroborated by local vibrational fingerprinting. By varying the type of functional groups attached at the graphene edge, we can now tune this nanoscale interface in a versatile manner and systematically study its effect on the ensuing physical and chemical properties.

\section{Experimental}

\section{GrEdge devices}

$\mathrm{Ti} / \mathrm{Pt}$ (50 nm/10 $\mathrm{nm}$ ) electrode lines were pre-patterned on $\mathrm{Si}$ with $\mathrm{SiO}_{2}$ (thickness of $500 \mathrm{~nm}$ ) with 4-micron gaps using photolithography. The chips were cleaned in a piranha solution $\left(\mathrm{H}_{2} \mathrm{SO}_{4}: \mathrm{H}_{2} \mathrm{O}_{2}\right)(3: 1)$ for 1 minute, and then in an oxygen plasma cleaner for 2 minutes before transfer of graphene. CVD grown-graphene-on-copper was cut into rectangular pieces (typically $2 \mathrm{~cm} \times 2 \mathrm{~cm}$ ) and a solution of poly(styrene) (PS)
(50 $\mathrm{mg} \mathrm{mL}^{-1}$ in toluene) was spotted over the CVD graphene foil as a polymer support and dried at $75{ }^{\circ} \mathrm{C}$ for 10 minutes. Then, the underlying copper was removed by etching in a solution of hydrochloric acid with added hydrogen peroxide $\left(1.4 \mathrm{~mol} \mathrm{~L}^{-1}\right.$ $\mathrm{HCl}+0.5 \mathrm{~mol} \mathrm{~L}^{-1} \mathrm{H}_{2} \mathrm{O}_{2}$ ) for 10 minutes. The PS/graphene slab was transferred to the $\mathrm{Si} / \mathrm{SiO}_{2}$ chips with the pre-patterned $\mathrm{Ti} / \mathrm{Pt}$ electrode lines and baked in an oven at $95{ }^{\circ} \mathrm{C}$ for 10 minutes before removal of PS in toluene ( 5 minutes with mechanical stirring). Finally, $\mathrm{Si} / \mathrm{SiO}_{2} /$ graphene chips were annealed at $600{ }^{\circ} \mathrm{C}$ under nitrogen atmosphere for 1 minute to improve the adhesion of graphene onto the silicon chips and remove possible residual organic contamination from the surface of the electrode. Prior to patterning of GrEdge electrodes, the removal of reminiscent copper particles was carried out by electrochemical etching (e-etching) in $\mathrm{HCl} 0.1 \mathrm{~mol} \mathrm{~L}^{-1}, 15$ scans in the potential range from $-1 \mathrm{~V}$ to $+0.1 \mathrm{~V}$ ( $v s$. $\mathrm{Ag} / \mathrm{AgCl}$ ) at $50 \mathrm{mV} \mathrm{s}^{-1}$. The patterning of GrEdge was also done by photolithography (using a Maskless Aligner MLA-100, Heidelberg Instruments) followed by oxygen plasma etching. Specifically, a polymer layer of the commercial positive photoresist S1805 (Microposit) was spin-coated on top of the sample ( $\sim 500 \mathrm{~nm}$ thickness), prebaked for 2:20 minutes at $90{ }^{\circ} \mathrm{C}$ and the negative of the desired area was exposed to light $(\lambda=365 \mathrm{~nm})$. The photoresist from the exposed areas was removed by development using mf319 developer (Microposit). The electrodes including contacted graphene are hence protected, with graphene being half protected and half exposed, separated by a sharp edge of the resist. The GrEdge was then obtained by exposure of non-protected graphene to oxygen plasma etching. For Raman spectroscopy and AFM imaging, the remaining photoresist was removed in acetone for 15 seconds.

\section{Electrochemical setup}

Electrochemical modification was carried out using a threeelectrode system comprising of GrEdge as working electrode, platinum wire $(\Phi=0.5 \mathrm{~mm})$ as the auxiliary electrode and $\mathrm{Ag} /$ $\mathrm{AgCl}$ as the reference electrode. The experiments were performed in an electrochemical cell with $20 \mathrm{~mL}$ of solution using an Ivium Compacstat potentiostat.

\section{Electrochemical modification of GrEdge with AuNPs}

The electrochemical modification was performed in the same electrochemical setup described above. GrEdge-AuNPs were obtained potentiostatically in four deposition steps as following: $0.4 \mathrm{~V}$ for $20 \mathrm{~s},-0.5 \mathrm{~V}$ for $5 \mathrm{~s}, 0.4 \mathrm{~V}$ for $20 \mathrm{~s}$ and $-0.5 \mathrm{~V}$ for $5 \mathrm{~s}$ (Fig. S3†). For GrEdge, the concentration of aqueous solution of gold metal salt and the background electrolyte for all the depositions were $10 \mu \mathrm{mol} \mathrm{L}^{-1} \mathrm{KAuCl}_{4}$ and $\mathrm{LiClO}_{4}$ $0.1 \mathrm{~mol} \mathrm{~L}^{-1}$.

\section{Electrochemical modification of GrEdge with pABA followed by AuNPs}

For the functionalization of GrEdge with pABA, 4-aminobenzylamine (4-ABA) aqueous solution at a concentration of $5 \mathrm{mmol} \mathrm{L}{ }^{-1}$ with $0.1 \mathrm{~mol} \mathrm{~L}{ }^{-1} \mathrm{LiClO}_{4}$ was polymerized using cyclic voltammetry at a potential range of $-0.2 \mathrm{~V}$ to $0.8 \mathrm{~V} v \mathrm{~s}$. $\mathrm{Ag} /$ 
$\mathrm{AgCl}$ for two cycles. Then, AuNPs arrays were deposited by chronoamperometry as described above by the use of $30 \mu \mathrm{mol}$ $\mathrm{L}^{-1} \mathrm{KAuCl}_{4}$ in $0.1 \mathrm{~mol} \mathrm{~L}^{-1} \mathrm{LiClO}_{4}$. The gold salt solution was always prepared right before the electrochemical deposition experiments.

\section{Raman spectroscopy and AFM images}

Raman spectra for GrEdge-AuNP electrodes were obtained on LabRam HR 800 equipped with a $1024 \times 256$ CCD detector (symphony; liquid $\mathrm{N}_{2}$ cooled), a $300 \mathrm{~L} \mathrm{~mm}^{-1}$ grating, a HeNe laser with an excitation wavelength of $632.8 \mathrm{~nm}$, a $100 \times(\mathrm{NA}$ 0.90) objective, and a detection pinhole of $100 \mu \mathrm{m}$. The power was maintained at $4.5 \mathrm{~mW}$ for acquisitions on all the graphene samples. Raman spectra for GrEdge-pABA-AuNPs were obtained on a JASCO NRS-4100 Raman spectrometer equipped with a $1650 \times 256 \mathrm{CCD}$ detector (Andor; air/Peltier-cooled, operating temperature: $-60{ }^{\circ} \mathrm{C}$ ), a $400 \mathrm{~L} \mathrm{~mm}^{-1}$ grating, a diode laser with an excitation of $632.8 \mathrm{~nm}$, and a $100 \times(\mathrm{NA}$ 0.90 ) objective and a power of $3.4 \mathrm{~mW}$. The Raman spectra and maps were recorded using LabSpec 6 for GrEdge-AuNPs electrodes and spectra manager for GrEdge-pABA-AuNPs. The spectrometer for both instruments was calibrated by determining the wavenumber position of Raman line of a silicon substrate at $520.7 \mathrm{~cm}^{-1}$ before data acquisition. Raman spectra and map images were processed using Origin 9.1. The Raman data was fitted with Lorentzian profile in Origin 9.1 or Mathematica to determine the peak position and FWHM. Raman maps were obtained by integrating the intensity in the range of $\pm 10 \mathrm{~cm}^{-1}$ around the main peak. AFM images were obtained using a commercial Dimension IV working in tapping mode in the ambient. AFM images were processed using Gwyddion.

\section{Scanning electron microscopy (SEM) and energy dispersive X- ray spectroscopy (EDX)}

SEM and EDX data were acquired using a JEOL JCM-6000 Neoscope ESEM-EDX instrument in high-vacuum mode (approx. 10-4 mbar). No coating or any other pretreatment was applied to the samples. The image shown in Fig. 1(d) is a secondary electron image, while for EDX, a rectangular area along the aligned nanoparticles was selected on a backscattered electron image for accumulation of the spectrum shown in Fig. S4, ESI. $\dagger$

\section{Conflicts of interest}

There are no conflicts to declare.

\section{Acknowledgements}

AY thanks the funding from the German Science Foundation (DFG) via the Graduate School of Analytical Sciences Adlershof (SALSA GSC1013). RMI acknowledges CAPES/AvH-Foundation (Iost-CAPES-2017-18) for financial support.

\section{References}

1 A. K. Geim and K. S. Novoselov, Nat. Mater., 2007, 6, 183-191.

2 A. K. Geim, Science, 2009, 324, 1530.

3 K. S. Novoselov, A. K. Geim, S. V. Morozov, D. Jiang, Y. Zhang, S. V. Dubonos, I. V. Grigorieva and A. A. Firsov, Science, 2004, 306, 666.

4 F. Schedin, A. K. Geim, S. V. Morozov, E. W. Hill, P. Blake, M. I. Katsnelson and K. S. Novoselov, Nat. Mater., 2007, 6, 652.

5 Q. H. Wang, Z. Jin, K. K. Kim, A. J. Hilmer, G. L. C. Paulus, C.-J. Shih, M.-H. Ham, J. D. Sanchez-Yamagishi, K. Watanabe, T. Taniguchi, J. Kong, P. Jarillo-Herrero and M. S. Strano, Nat. Chem., 2012, 4, 724.

6 K. Yokota, K. Takai and T. Enoki, Nano Lett., 2011, 11, 36693675.

7 Z. Yan, Z. Sun, W. Lu, J. Yao, Y. Zhu and J. M. Tour, ACS Nano, 2011, 5, 1535-1540.

8 C. N. R. Rao, A. K. Sood, K. S. Subrahmanyam and A. Govindaraj, Angew. Chem., Int. Ed., 2009, 48, 7752-7777.

9 E. P. Randviir, D. A. C. Brownson and C. E. Banks, Mater. Today, 2014, 17, 426-432.

10 J. Greenwood, T. H. Phan, Y. Fujita, Z. Li, O. Ivasenko, W. Vanderlinden, H. Van Gorp, W. Frederickx, G. Lu, K. Tahara, Y. Tobe, I. H. Uji, S. F. Mertens and S. De Feyter, ACS Nano, 2015, 9, 5520-5535.

11 T. Kuila, S. Bose, A. K. Mishra, P. Khanra, N. H. Kim and J. H. Lee, Prog. Mater. Sci., 2012, 57, 1061-1105.

12 D. W. Boukhvalov and M. I. Katsnelson, J. Phys.: Condens. Matter, 2009, 21, 344205.

13 A. Hirsch, J. M. Englert and F. Hauke, Acc. Chem. Res., 2013, 46, 87-96.

14 C. K. Chua and M. Pumera, Chem. Soc. Rev., 2013, 42, 3222-3233. 15 X. Wang and H. Dai, Nat. Chem., 2010, 2, 661-665.

16 M. Y. Han, B. Ozyilmaz, Y. Zhang and P. Kim, Phys. Rev. Lett., 2007, 98, 206805.

17 X. Jia, M. Hofmann, V. Meunier, B. G. Sumpter, J. CamposDelgado, J. M. Romo-Herrera, H. Son, Y.-P. Hsieh, A. Reina, J. Kong, M. Terrones and M. S. Dresselhaus, Science, 2009, 323, 1701.

18 A. Muge and J. C. Yves, Jpn. J. Appl. Phys., 2011, 50, 070101. 19 K. A. Ritter and J. W. Lyding, Nat. Mater., 2009, 8, 235-242. 20 O. Hod, V. Barone, J. E. Peralta and G. E. Scuseria, Nano Lett., 2007, 7, 2295-2299.

21 W. Yuan, Y. Zhou, Y. Li, C. Li, H. Peng, J. Zhang, Z. Liu, L. Dai and G. Shi, Sci. Rep., 2013, 3, 2248.

22 C. Casiraghi, A. Hartschuh, H. Qian, S. Piscanec, C. Georgi, A. Fasoli, K. S. Novoselov, D. M. Basko and A. C. Ferrari, Nano Lett., 2009, 9, 1433-1441.

23 A. K. Gupta, T. J. Russin, H. R. Gutierrez and P. C. Eklund, ACS Nano, 2009, 3, 45-52.

24 Y. F. Kobayashi, K.-I. Fukui and T. Enoki, Phys. Rev. B: Condens. Matter Mater. Phys., 2006, 73, 125415.

25 K. Suenaga and M. Koshino, Nature, 2010, 468, 1088-1090.

26 Z. Liu, K. Suenaga, P. J. Harris and S. Iijima, Phys. Rev. Lett., 2009, 102, 015501. 
27 F. Cervantes-Sodi, G. Csányi, S. Piscanec and A. C. Ferrari, Phys. Rev. B: Condens. Matter Mater. Phys., 2008, 77, 165427.

28 Z. Xiang, Q. Dai, J. F. Chen and L. Dai, Adv. Mater., 2016, 28, 6253-6261.

29 A. Shen, Y. Zou, Q. Wang, A. W. Dryfe Robert, X. Huang, S. Dou, L. Dai and S. Wang, Angew. Chem., Int. Ed., 2014, 53, 10804-10808.

30 I.-Y. Jeon, H.-J. Choi, S.-M. Jung, J.-M. Seo, M.-J. Kim, L. Dai and J.-B. Baek, J. Am. Chem. Soc., 2013, 135, 1386-1393.

31 J. Xu, J. Shui, J. Wang, M. Wang, H.-K. Liu, S. X. Dou, I.-Y. Jeon, J.-M. Seo, J.-B. Baek and L. Dai, ACS Nano, 2014, 8, 10920-10930.

32 M. Quintana, A. Montellano, A. E. del Rio Castillo, G. V. Tendeloo, C. Bittencourt and M. Prato, Chem. Commun., 2011, 47, 9330-9332.

33 Y. He, M. Garnica, F. Bischoff, J. Ducke, M. L. Bocquet, M. Batzill, W. Auwarter and J. V. Barth, Nat. Chem., 2017, 9, 33-38.

34 S. Wickenburg, J. Lu, J. Lischner, H. Z. Tsai, A. A. Omrani, A. Riss, C. Karrasch, A. Bradley, H. S. Jung, R. Khajeh, D. Wong, K. Watanabe, T. Taniguchi, A. Zettl, A. H. Neto, S. G. Louie and M. F. Crommie, Nat. Commun., 2016, 7, 13553. 35 X. Li, W. Cai, J. An, S. Kim, J. Nah, D. Yang, R. Piner, A. Velamakanni, I. Jung, E. Tutuc, S. K. Banerjee, L. Colombo and R. S. Ruoff, Science, 2009, 324, 1312.

36 J. Song, F.-Y. Kam, R.-Q. Png, W.-L. Seah, J.-M. Zhuo, G.-K. Lim, P. K. H. Ho and L.-L. Chua, Nat. Nanotechnol., 2013, 8, 356.

37 M. Iost Rodrigo, N. Crespilho Frank, L. Zuccaro, K. Yu Hak, M. Wodtke Alec, K. Kern and K. Balasubramanian, ChemElectroChem, 2014, 1, 2070-2074.

38 K. Balasubramanian, L. Zuccaro and K. Kern, Adv. Funct. Mater., 2014, 24, 6348-6358.

39 K. Balasubramanian and M. Burghard, J. Mater. Chem., 2008, 18, 3071-3083.

40 M. Scolari, A. Mews, N. Fu, A. Myalitsin, T. Assmus, K. Balasubramanian, M. Burghard and K. Kern, J. Phys. Chem. C, 2008, 112, 391-396.

41 F. Schedin, E. Lidorikis, A. Lombardo, V. G. Kravets, A. K. Geim, A. N. Grigorenko, K. S. Novoselov and A. C. Ferrari, ACS Nano, 2010, 4, 5617-5626.

42 L. M. Malard, M. A. Pimenta, G. Dresselhaus and M. S. Dresselhaus, Phys. Rep., 2009, 473, 51-87.

43 Y. You, Z. Ni, T. Yu and Z. Shen, Appl. Phys. Lett., 2008, 93, 163112.

44 M. Begliarbekov, O. Sul, S. Kalliakos, E.-H. Yang and S. Strauf, Appl. Phys. Lett., 2010, 97, 031908.

45 A. C. Ferrari and D. M. Basko, Nat. Nanotechnol., 2013, 8, 235-246.
46 L. Zuccaro, K. Kern and K. Balasubramanian, ACS Nano, 2015, 9, 3314-3323.

47 K. Kneipp, H. Kneipp, I. Itzkan, R. R. Dasari and M. S. Feld, Chem. Rev., 1999, 99, 2957-2976.

48 D. M. Basko, S. Piscanec and A. C. Ferrari, Phys. Rev. B: Condens. Matter Mater. Phys., 2009, 80, 165413.

49 S. Reich and C. Thomsen, Philos. Trans. R. Soc., A, 2004, 362, 2271-2288.

50 A. C. Ferrari, J. C. Meyer, V. Scardaci, C. Casiraghi, M. Lazzeri, F. Mauri, S. Piscanec, D. Jiang, K. S. Novoselov, S. Roth and A. K. Geim, Phys. Rev. Lett., 2006, 97, 187401.

51 R. Yang, Z. W. Shi, L. C. Zhang, D. X. Shi and G. Y. Zhang, Nano Lett., 2011, 11, 4083-4088.

52 D. C. Elias, R. R. Nair, T. M. G. Mohiuddin, S. V. Morozov, P. Blake, M. P. Halsall, A. C. Ferrari, D. W. Boukhvalov, M. I. Katsnelson, A. K. Geim and K. S. Novoselov, Science, 2009, 323, 610-613.

53 H. Sahin, O. Leenaerts, S. K. Singh and F. M. Peeters, Wiley Interdiscip. Rev.: Comput. Mol. Sci., 2015, 5, 255-272.

54 Y. Wang, X. F. Xu, J. Lu, M. Lin, Q. L. Bao, B. Ozyilmaz and K. P. Loh, ACS Nano, 2010, 4, 6146-6152.

55 C. L. Haynes and R. P. Van Duyne, J. Phys. Chem. B, 2003, 107, 7426-7433.

56 A. Bottcher, M. Heil, N. Sturzl, S. S. Jester, S. Malik, F. PerezWillard, P. Brenner, D. Gerthsen and M. M. Kappes, Nanotechnology, 2006, 17, 5889-5894.

57 Y. Yi, G. Weinberg, M. Prenzel, M. Greiner, S. Heumann, S. Becker and R. Schlögl, Catal. Today, 2017, 295, 32-40.

58 J. Heinze, B. A. Frontana-Uribe and S. Ludwigs, Chem. Rev., 2010, 110, 4724-4771.

59 K. Balasubramanian, M. Friedrich, C. Jiang, Y. Fan, A. Mews, M. Burghard and K. Kern, Adv. Mater., 2003, 15, 1515-1518.

60 L. Zuccaro, J. Krieg, A. Desideri, K. Kern and K. Balasubramanian, Sci. Rep., 2015, 5, 11794.

61 L. J. A. Macedo, F. C. D. A. Lima, R. G. Amorim, R. O. Freitas, A. Yadav, R. M. Iost, K. Balasubramanian and F. N. Crespilho, Nanoscale, 2018, 10, 15048-15057.

62 M. Cochet, G. Louarn, S. Quillard, M. I. Boyer, J. P. Buisson and S. Lefrant, J. Raman Spectrosc., 2000, 31, 1029-1039.

63 M. Cochet, G. Louarn, S. Quillard, J. P. Buisson and S. Lefrant, J. Raman Spectrosc., 2000, 31, 1041-1049.

64 J. B. Lambert, H. F. Shurvell, D. A. Lightner and R. G. Cooks, Introduction to organic spectroscopy, Macmillan Publishing Company, 1987.

65 M. C. Bernard and A. Hugot-Le Goff, Electrochim. Acta, 2006, 52, 595-603.

66 M.-C. Bernard, S. Cordoba de Torresi and A. H.-L. Goff, Electrochim. Acta, 1999, 44, 1989-1997. 